\title{
Reduced Respiratory Capacity in Muscle Mitochondria of Obese Subjects
}

\author{
Linda Bakkman ${ }^{a, b} \quad$ Maria Fernström ${ }^{c}$ Peter Loogna ${ }^{d}$ Olav Rooyackers ${ }^{c}$ Lena Brandt ${ }^{a}$ \\ Ylva Trolle Lagerros ${ }^{a, b}$ \\ a Unit of Clinical Epidemiology, Department of Medicine, Karolinska Institutet Solna, \\ ${ }^{b}$ Obesity Unit, Department of Medicine, \\ ${ }^{c}$ Department of Anaesthesiology and Intensive Care, Karolinska Institutet Huddinge, \\ ${ }^{\mathrm{d}}$ Bariatric Center, Sophiahemmet, Stockholm, Sweden
}

\section{Keywords \\ Metabolism - Obesity · Resting energy expenditure · \\ Thermogenesis - Muscle mitochondria}

\section{Summary}

Background/Aims: The extent of weight gain varies among individuals despite equal calorie overconsumption. Furthermore, weight gain is often less than expected from energy excess. This suggests differences in metabolic efficiency and basal metabolism. Since mitochondrial uncoupling accounts for a substantial portion of the basal metabolic rate, we compared skeletal muscle mitochondrial respiration in obese subjects to normal-weight reference groups with various degrees of physical activity. Methods: Muscle biopsies were taken from the vastus lateralis muscle of 9 healthy obese subjects (BMI $40 \pm 3$ ). Mitochondria were isolated and analyzed for coupled (state 3 ) and uncoupled (state 4) respirations as well as mitochondrial efficiency ( $\mathrm{P} / \mathrm{O}$ ratio) using pyruvate as a substrate. Respiratory data were compared to reference groups $A$, normal-weight untrained (BMI $24 \pm 0.7)$, and $B$, normal-weight trained (BMI $24 \pm 0.6$ ). Results: Obese subjects had a decreased respiratory capacity per mitochondrial volume compared to the reference groups: this was evident in state $4(65 \%$ and $35 \%$ of reference group $A$ and $B$, respectively) and state $3(53 \%$ and $29 \%$ of $A$ and $B$, respectively) ( $p<0.05)$. Conclusion: Obese subjects had a low capacity for fuel oxidation, which may play a role in the predisposition of obesity. However, whether lower mitochondrial capacity is a cause or a consequence of obesity requires further research.

\section{Introduction}

Obesity is a result of a prolonged imbalance between energy intake and energy expenditure. Nevertheless, several studies have shown an interindividual variability in the susceptibility to weight gain, in response to controlled overconsumption of calories [1]. In addition, experiments with overfeeding have shown that weight gain is often less than expected from the energy excess $[2,3]$, suggesting alterations in metabolic efficiency and the basal metabolism.

For most people, basal metabolism is by far the largest contributor to the body's energy expenditure [4]. Although the skeletal muscle has a low basal metabolism per unit of weight, it can constitute up to $30 \%$ of the body's oxygen consumption at rest [5], making the muscle an obvious target to study metabolism in relation to obesity.

Mitochondria are essential for energy production at the cellular level. Therefore, differences in energy expenditure and basal metabolism may be attributed to mitochondrial function. Mitochondrial dysfunction is now considered a key factor in the development of insulin resistance [6], which is strongly associated with obesity. On the other hand, Fleischman et al. [7] have dismissed an association between obesity and mitochondrial dysfunction based on comparisons in muscle ATP synthesis between overweight and normal-weight children.

Proton leakage of the inner mitochondrial membrane (uncoupling) results in metabolic inefficiency - where part of the energy diffuses as heat rather than being consumed or stored as fat. Uncoupling accounts for a substantial portion of basal metabolic rate [8] and thus becomes crucial for energy and weight control. When the coupling between mitochondrial oxidation and ATP synthesis is reduced, the energy will dissipate as heat instead of being available for consumption and

\section{KARGER}

Fax +49 7614520714

Information@Karger.de

www.karger.com
(ㄷ) 2010 S. Karger GmbH, Freiburg

Accessible online at:

www.karger.com/ofa 
storage (fat mass). This potential for metabolic adjustment, called the adaptive thermogenesis [6], may serve as an important factor in preventing obesity through increased expenditure in subjects on hypercaloric diets.

It has been shown that obese individuals exhibit reduced levels of oxidative enzymes and lower mitochondrial volume compared to normal-weight individuals [9-12]. Furthermore, at least one study has shown a decreased activity of the electron transport chain (ETC) in muscle mitochondria from obese subjects compared to lean control subjects after adjusting for mitochondrial volume [10]. Therefore, we hypothesize that less proton leakage, in other words more efficient mitochondria, might lead to less oxygen consumption and thereby an increased risk of weight gain.

Thus, the aim of the present study was to examine if mitochondrial respiratory capacity in skeletal muscle of obese subjects is reduced compared to their lean counterparts.

\section{Participants and Methods}

\section{Subjects}

Nine consecutive healthy obese subjects, who were referred to obesity surgery at the Bariatric Center, Sophiahemmet, Stockholm, during spring 2009, participated in the study. Exclusion criteria were smoking and any type of medication, resulting in a selection of only women.

All subjects were informed about the study and provided a written informed consent. The subjects were also asked to self-report their total physical activity level - all reported an inactive or low active lifestyle. In addition, subjects were asked to wear electronic pedometers (Silva, Ex1 Distance) during 3 consecutive days and to keep a record of steps taken. The study was approved by the Ethics Committee of the Karolinska Institutet, Stockholm, Sweden.

\section{Reference Groups}

The normal-weight reference groups represented subjects in two separate studies that have previously been conducted by our group. Reference group A, normal-weight untrained, consisted of 9 normal-weight individuals (4 women, 5 men) not exercising regularly [13] and reference group $\mathrm{B}$, normal-weight trained, consisted of 9 ultra-endurance performance athletes, all men [14]. Maximal oxygen consumption $\left(\mathrm{VO}_{2}-\mathrm{max}\right)$ was used to assess fitness level. Characteristics of the study subjects and reference groups are described in table 1.

\section{Surgery}

All obese subjects underwent a laparoscopic gastric Roux-en-Y bypass (RYGB). RYGB is a surgical procedure leading to caloric restriction by both a restrictive (stomach volume reduction) and malabsorptive (bypassing of the duodenum and a part of the jejunum) mechanism. An antecolic, antegastric gastrojejunostomy was performed. The surgery resulted in an alimentary limb of $120 \mathrm{~cm}$ and a biliopancreatic limb of $60 \mathrm{~cm}$. In addition, the gastric pouch was decreased to about $20-30 \mathrm{ml}$ in size.

\section{Muscle Biopsies and Isolation of Mitochondria}

Muscle biopsies were obtained by a needle biopsy technique from the vastus lateralis muscle immediately after induction of anesthesia, but before the gastric bypass. An incision was made through the skin and fascia by a scalpel under general and local anesthesia (5-10 ml Carbocain, $20 \mathrm{mg} / \mathrm{ml}$ ). The biopsy was taken using the technique of Bergstrom [15]. On average, a $72 \mathrm{mg}$ muscle sample was used for isolation of mitochondria.
Table 1. Characteristics of subjects and reference groups in the study of mitochondrial function in obese subjects compared to normal-weight individuals with various degrees of fitness levels

\begin{tabular}{llll}
\hline & \multicolumn{2}{l}{ Reference group } & $\begin{array}{l}\text { Obese subjects } \\
(\mathrm{n}=9)\end{array}$ \\
\cline { 2 - 3 } & $\mathrm{A}(\mathrm{n}=9)$ & $\mathrm{B}(\mathrm{n}=9)$ & \\
\hline Degree of fitness level & untrained & trained & untrained \\
Age, years & $25 \pm 1.0$ & $27 \pm 0.9$ & $36 \pm 2.0$ \\
BMI, kg/m ${ }^{2}$ & $24 \pm 0.7$ & $24 \pm 0.6$ & $40 \pm 3.0$ \\
Currently smoking & no & no & no \\
\hline
\end{tabular}

To isolate the mitochondria we used the technique of Tonkonogi and Sahlin [16]. Briefly, muscle specimens were disintegrated mechanically with scissors and treated with $0.4 \mathrm{mg} / \mathrm{ml}$ protease (Sigma P-4789) for chemical disruption. This was followed by homogenization and differential centrifugation to separate mitochondria from other cell organelles. The final mitochondria pellet was resuspended in a preserving medium ( $225 \mathrm{mmol} / 1 \mathrm{mannitol}, 75 \mathrm{mmol} / 1$ sucrose, $10 \mathrm{mmol} / 1$ Tris-base, $0.1 \mathrm{mmol} / \mathrm{l}$ EDTA, and $0.2 \%$ bovine serum albumin, $\mathrm{pH}$ 7.4). Immediately after receiving the mitochondrial suspension the analysis of respiratory activity was performed.

\section{Analysis of Mitochondrial Respiration}

Mitochondrial respiratory activity was measured using a Clark-type electrode (Hansatech DW1; Hansatech, King's Lynn, Norfolk, UK) at $25{ }^{\circ} \mathrm{C}$. Respiration was analyzed in an oxygraph medium (225 mmol/l mannitol, $75 \mathrm{mmol} / \mathrm{l}$ sucrose, $10 \mathrm{mmol} / 1$ Tris-base, $10 \mathrm{mmol} / 1 \mathrm{~K}_{2} \mathrm{HPO}_{4}, 0.1 \mathrm{mmol} / \mathrm{l}$ EDTA, $80 \mu \mathrm{mol} / 1 \mathrm{MgCl}_{2}$, and $0.2 \%$ bovine serum albumin, $5 \mathrm{mmol} / \mathrm{l}$ pyruvate, $2 \mathrm{mmol} / \mathrm{l}$ malate, $\mathrm{pH}$ 7.1). The mitochondrial suspension was then added to the reaction medium.

Coupled respiration (state 3), i.e. production of ATP, was initiated by the addition of ADP (final concentration $0.3 \mathrm{mmol} / \mathrm{l}$ ), which in turn increases the oxygen consumption of the mitochondria. The oxygen consumption does not cease completely even though all ADP phosphorylates to ATP. Instead, the respiratory rate returns to the level it had prior the ADP-addition, reflecting the thermogenesis (state 4, uncoupled respiration).

The respiratory control index (RCI) was calculated as the ratio between state 3 and state 4 respirations. The efficiency of oxidative phosphorylation ( $\mathrm{P} / \mathrm{O}$ ratio) was calculated by dividing the amount of added, i.e. phosphorylated, ADP by the amount of oxygen consumed during state 3 [17]. Subsequent to measuring respiration, the remaining mitochondrial suspension was frozen and stored at $-80{ }^{\circ} \mathrm{C}$. Defrosted samples were then used for spectrophotometrical determination of mitochondrial citrate synthase activity (CS) as described by Tonkonogi et al. [18].

\section{Statistics}

Comparisons of respiration data between the three groups (reference group A, reference group B, and obese subjects) were conducted using the non-parametric Kruskal-Wallis test. The location of significance was tested by a Wilcoxon signed-ranked test. The statistical analyses were performed using PC-SAS. The results are expressed as mean \pm SEM. Statistical significance was set at $\mathrm{p}<0.05$.

\section{Results}

The characteristics of the subjects and reference groups are shown in table 1 . The reference groups were of similar BMI (normal weight) and age, whereas the obese subjects were 


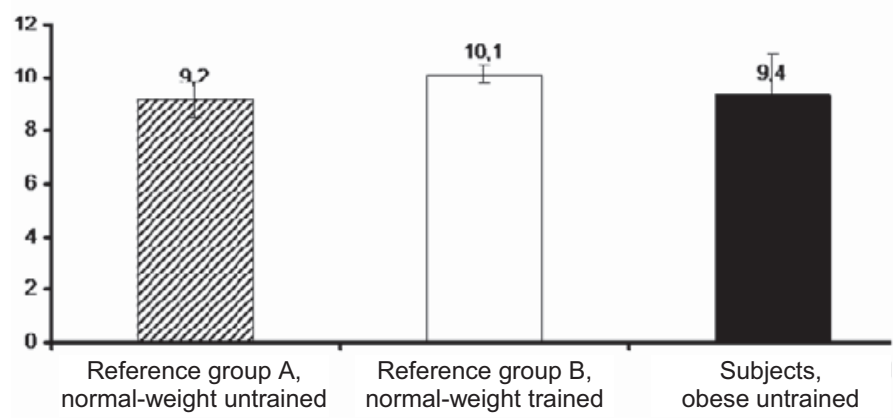

b

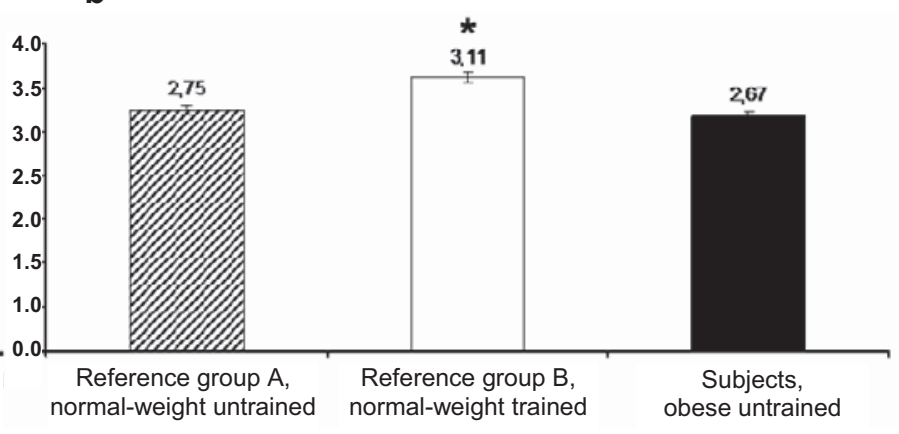

Fig. 1. Respiratory data on (a) respiratory control index (RCI) and (b) mitochondrial efficiency (P/O-ratio) in obese subjects compared to normalweight reference groups with various degrees of fitness levels. RCI was calculated as state 3/state 4 and P/O-ratio was calculated as the ratio between added ADP and consumed oxygen during respiration with pyruvate. Values are mean \pm SEM from 9 subjects. *p $<0.05$ compared with reference group A, normal-weight untrained.

a

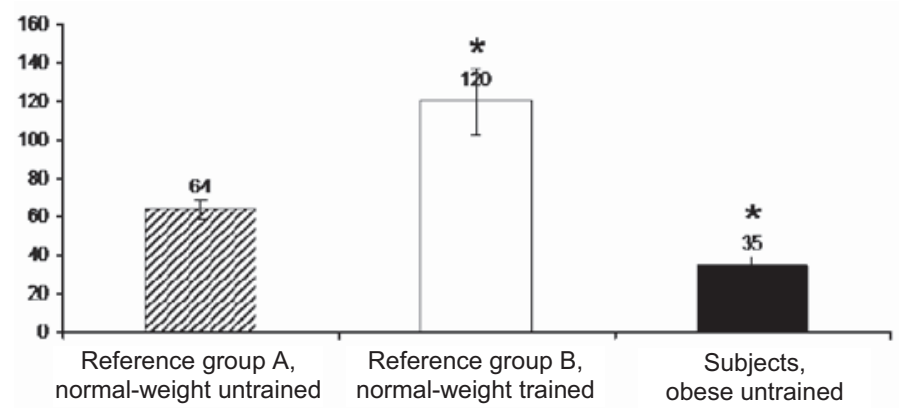

b

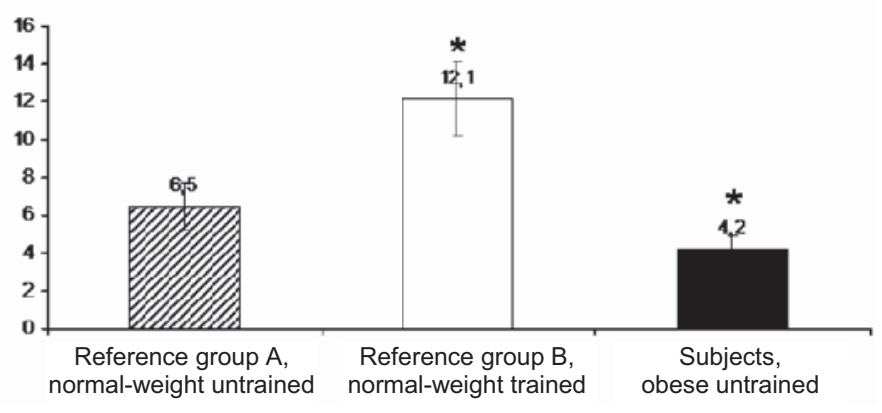

Fig. 2. Respiratory data on (a) coupled (state 3) and (b) uncoupled (state 4) respiration in obese subjects compared to normal-weight reference groups with various degrees of fitness levels. Unit; nmol $\mathrm{O}_{2} / \mathrm{min}^{-1} \mathrm{U} \mathrm{CS}^{-1}$. State 3 corresponds to maximal ADP stimulated respiration rate and state 4 to the respiration rate in the absence of ADP. Respiration was measured with pyruvate and expressed per unit of citrate synthase (CS) activity. Values are mean \pm SEM from 9 subjects. ${ }^{*} \mathrm{p}<0.05$ compared with reference group A, normal-weight untrained.

about 10 years older. The obese subjects had normal levels of conventional obesity-related metabolic risk factors (HbA1c; $4.7 \pm 0.12 \%$, triglycerides, TG; $1.6 \pm 0.23 \mathrm{mmol} / \mathrm{l}$, low-density lipoproteins, LDL; $2.8 \pm 0.27 \mathrm{mmol} / \mathrm{l}$, high-density lipoproteins, HDL; $1.0 \pm 0.13 \mathrm{mmol} / \mathrm{l})$.

Reference group A was defined as untrained based on their $\mathrm{VO}_{2}$-max of $3.62 \pm 0.27 \mathrm{l} / \mathrm{min}$. The trained reference group $\mathrm{B}$ was endurance athletes with a $\mathrm{VO}_{2}$-max of $5.01 \pm$ $0.14 \mathrm{l} / \mathrm{min}$. The obese subjects were considered untrained based on self-reported activity level and 3 days of objective measurements with pedometers. The subjects took on average $6,630 \pm 840$ steps per day.

Despite rough mechanical and enzymatic handling during the isolation procedure, the mitochondria were kept functionally intact. This was demonstrated by an RCI around 10 (fig. 1a) and P/O-ratio about 3 (fig. 1b). Neither RCI nor $\mathrm{P} / \mathrm{O}$-ratio were significantly different between the obese subjects and the lean, untrained reference group (A). However, mitochondrial efficiency, i.e. P/O-ratio, was significantly higher among the trained (B) than among the untrained reference group (A).
The rate of respiration in the presence of ADP (state 3) expressed per unit of CS (a marker of mitochondrial volume) was $45 \%$ lower among the obese subjects than in the normal-weight, untrained reference group (fig. 2), $\mathrm{p}=$ 0.0017 . The trained reference group had in turn an $88 \%$ higher state 3 respiration than the untrained reference group, $\mathrm{p}=0.0005$. In the uncoupled state (state 4 ), obese subjects exhibited respiration that was $65 \%$ of the uncoupling rate in the normal-weight, untrained reference group, $\mathrm{p}=0.0343$, and only $35 \%$ of the uncoupling rate measured among the trained, $\mathrm{p}=0.0005$.

\section{Discussion}

This article highlights differences in mitochondrial capacity in skeletal muscle between obese and normal-weight individuals. We found that obese subjects had a decreased respiratory capacity per mitochondrial volume compared to two normalweight reference groups with various degrees of fitness. This was evident in coupled isolated mitochondria (state 3 respira- 
tion) using pyruvate as substrate as well as in the uncoupled state (state 4). The results support a role of mitochondrial respiratory capacity in weight regulation and interindividual differences of susceptibility to obesity. A reduced uncoupling would make more of the consumed energy available which, if not expended, would eventually be stored as fat. However, it is difficult to state whether this decreased mitochondrial capacity and uncoupling is part of the cause or the consequence of obesity.

Our results raise new questions about the mechanism behind the reduced mitochondrial capacity. For instance, fibertype distribution has been proposed as an etiological factor in the development of obesity, since an inverse relationship between the fraction type I fiber and body fat has been observed [19]. Type I muscle fibers have a higher capillary density and lipid storage capacity than type II fibers [20], making them more suitable for fat oxidation. We did not measure fibertype distribution due to limited material, sometimes as low as $10 \mathrm{mg}$. Thus, it is possible that the decreased mitochondrial respiratory capacity among the obese subjects depended on a low percentage of type I fibers.

In fact, the decreased respiratory capacity may be reserved for the subsarcolemmal mitochondria (SS-M) fraction. SS-M have been postulated to be critical for substrate transport, signal transduction, and fatty acid oxidation [21]. As SS-M appear to be important for fat oxidation, it has been proposed that the reduced capacity of SS-M may contribute to the accumulation of intramyocellular lipids [22] leading to obesity. A disproportional reduction of SS-M activity might play a role in the pathogenesis of obesity.

Although there are a number of possible explanations for the reduced mitochondrial capacity, some explanations could, based on good grounds, be discarded. The lower rate of oxygen utilization, in both coupled and uncoupled respiration, was measured with pyruvate as substrate. It is possible that the outcome would have been different using fat as a substrate. It has been proposed that obese individuals often have a higher dietary fat intake than lean persons [23]. A higher fat intake would result in elevated levels of free fatty acids in the circulation. This may in turn lead to increased fat oxidation in mitochondria [24]. A higher capacity to oxidize fat, thus a higher relative fat oxidation at the expense of pyruvate, could be a plausible explanation for the impaired respiration rate among the obese subjects.

Conversely, there is considerable evidence against this theory. First, a reduction in CPT activity has been found among obese compared to lean counterparts [25]. This restricts the flow of fatty acids into the mitochondria for oxidation and will in turn promote lipid accumulation and lipogenesis. Second, Holloway et al. [26] found that even though oxidation of fatty acid is reduced at whole muscle level in obesity, the ability to oxidize fatty acids is not impaired at the mitochondrial level.

Third, the blood lipoprotein levels (measured as TG, LDL, and HDL) among our obese subjects were all within the nor- mal range. Finally, measurements of substrate utilization suggest that obese individuals exhibit reduced fat oxidation and a higher dependence of carbohydrates (measured as higher respiratory quotients) compared to lean individuals [27]. Consequently, these arguments contradict the fact that the reduced oxidation we observed would be limited to carbohydrates. In fact, it should be noted that, based on the motives described above, the oxidation among the obese subjects may have been even more reduced with fat as fuel.

We also find it unlikely that the reduced mitochondrial oxidative capacity solely reflects a more sedentary lifestyle among the obese. The pedometer-determined physical activity level among the obese subjects was 6,630 steps per day, thus not extremely low. Less than 5,000 steps/day is used as a 'sedentary lifestyle index' [28]. A 'low active' level corresponds to about 5,000-7,499 steps/day, which represents a typical activity level for most people not engaged in sports/ exercise [28]. When comparing the normal-weight reference groups with different degrees of physical activity, the mitochondrial efficiency $(\mathrm{P} / \mathrm{O}$ ratio) differed significantly. If the respiratory data only reflected differences in the degree of activity level we should, in similar ways, have seen a distinction in $\mathrm{P} / \mathrm{O}$ ratio between the obese subjects and the untrained reference group.

The obese subjects are about 10 years older than the normal-weight reference groups. It can therefore be argued that the reduced mitochondrial capacity is due to age differences. Whether or not age is associated with a reduction in skeletal muscle mitochondrial function is not entirely clear. We have previously rejected the possibility that the differences in respiration of isolated mitochondria are due to aging [29]. There were no differences to be found in respiration of isolated mitochondria from older compared to younger subjects [29]. However, there are conflicting data from an in vitro study showing a decreased state 3 respiration in isolated mitochondria from elderly subjects [30]. Additionally, in vivo results showed a reduced rate of mitochondrial oxidative activity by approximately $40 \%$ in old subjects compared to younger counterparts [31]. However, these differences were observed when age differences were considerable (more than 40 years) and are thus likely to be of minor importance in our study, where the difference between the obese subjects and the reference groups is only about 10 years. Likewise, a weakness of our study could be the comparison across gender. Ideally, the gender composition should have remained the same in all groups. We intended to include obese men as well. However, we found, like many others, that obese but otherwise healthy men eligible for gastric bypass are difficult to find.

On the other hand, we find no support in the literature of any gender differences in mitochondrial function. We therefore find it unlikely that this weakness affected the results.

Several studies have implicated that not only does a lower mitochondrial content play a role in the etiology of obesity, 
but so does also an impaired mitochondrial capability $[6,10]$. However, the evidence for a reduced mitochondrial function in obesity is still poor. To our knowledge, these are the first data comparing coupled and uncoupled respiration in human isolated mitochondria between obese and normal-weight subjects. The finding of a lower mitochondrial capacity among the obese may have important implications for the understanding of human variability in metabolic efficiency and body weight regulation. More studies are needed to illuminate the mechanisms behind these findings.

\section{Conclusion}

We report a decreased mitochondrial oxidative capacity in skeletal muscle of obese subjects. A low capacity for fuel oxidation could play a role in the predisposition in obesity. It remains to be established whether the reduced oxidative capacity is a cause or consequence of obesity. To study whether mitochondrial function is normalized by weight loss, without change in physical activity, might answer this question and appears to be an important area of research.

\section{Acknowledgements}

This study was primarily funded by Banverket's research fund, Sweden. Mitochondrial analyses were performed with a grant from the Swedish Research Council (no: 14244). The pedometers were generously provided by Abbott Scandinavia AB. We express our appreciation to Susanne Rantakyrö at Bariatric Center for her efforts coordinating the biopsy sampling. We also acknowledge our colleague Towe Jacobsson for laboratory assistance. Finally, we greatly appreciate the research volunteers who participated in the study.

\section{Disclosure}

The authors declare no conflicts of interest.

\section{References}

1 Sims EA, Danforth E Jr, Horton ES, Bray GA, Glennon JA, Salans LB: Endocrine and metabolic effects of experimental obesity in man. Recent Prog Horm Res 1973;29:457-496.

$\checkmark 2$ Goele K, Bosy-Westphal A, Rumcker B, Lagerpusch M, Muller MJ: Influence of changes in body composition and adaptive thermogenesis on the difference between measured and predicted weight loss in obese women. Obes Facts 2009;2:105-109.

-3 Joosen AM, Westerterp KR: Energy expenditure during overfeeding. Nutr Metab (Lond) 2006;3:25.

4 McArdle W: Exercise Physiology, ed 2. Philadelphia, Lea \& Febiger, 1986.

5 Zurlo F, Larson K, Bogardus C, Ravussin E: Skeletal muscle metabolism is a major determinant of resting energy expenditure. J Clin Invest 1990;86: 1423-1427.

6 Lowell BB, Shulman GI: Mitochondrial dysfunction and type 2 diabetes. Science 2005;307:384-387.

$\checkmark 7$ Fleischman A, Kron M, Systrom DM, Hrovat M, Grinspoon SK: Mitochondrial function and insulin resistance in overweight and normal-weight children. J Clin Endocrinol Metab 2009;94:4923-4930.

$>8$ Rolfe DF, Brand MD: Contribution of mitochondrial proton leak to skeletal muscle respiration and to standard metabolic rate. Am J Physiol 1996;271: C1380-1389.

9 Bass A, Vondra K, Rath R, Vitek V, Havranek $\mathrm{T}$ : Metabolic changes in the quadriceps femoris muscle of obese people. Enzyme activity patterns of energy-supplying metabolism. Pflugers Arch 1975 359:325-334.

10 Kelley DE, He J, Menshikova EV, Ritov VB: Dysfunction of mitochondria in human skeletal muscle in type 2 diabetes. Diabetes 2002;51:2944-2950.

11 Kim JY, Hickner RC, Cortright RL, Dohm GL, Houmard JA: Lipid oxidation is reduced in obese human skeletal muscle. Am J Physiol Endocrinol Metab 2000;279:E1039-1044.
12 Simoneau JA, Colberg SR, Thaete FL, Kelley DE: Skeletal muscle glycolytic and oxidative enzyme capacities are determinants of insulin sensitivity and muscle composition in obese women. Faseb $\mathbf{J}$ 1995;9:273-278.

13 Fernstrom M, Tonkonogi M, Sahlin K: Effects of acute and chronic endurance exercise on mitochondrial uncoupling in human skeletal muscle. J Physiol 2004;554:755-763.

14 Fernstrom M, Bakkman L, Tonkonogi M, Shabalina IG, Rozhdestvenskaya Z, Mattsson CM, Enqvist JK, Ekblom B, Sahlin K: Reduced efficiency, but increased fat oxidation, in mitochondria from human skeletal muscle after 24-h ultraendurance exercise. J Appl Physiol 2007;102:1844-1849.

15 Bergstrom J: Percutaneous needle biopsy of skeletal muscle in physiological and clinical research. Scand J Clin Lab Invest 1975;35:609-616.

16 Tonkonogi M, Sahlin K: Rate of oxidative phosphorylation in isolated mitochondria from human skeletal muscle: effect of training status. Acta Physiol Scand 1997;161:345-353.

17 Chance B, Williams GR: Respiratory enzymes in oxidative phosphorylation. I. Kinetics of oxygen utilization. J Biol Chem 1955;217:383-393.

18 Tonkonogi M, Harris B, Sahlin K: Increased activity of citrate synthase in human skeletal muscle after a single bout of prolonged exercise. Acta Physiol Scand 1997;161:435-436.

19 Wade AJ, Marbut MM, Round JM: Muscle fibre type and aetiology of obesity. Lancet 1990;335:805808.

20 Saltin B, Gollnick PD: Handbook of physiology. Skeletal muscle. Bethesda, MD, American Physiological Society, 1983.

21 Hood DA: Invited review: Contractile activity-induced mitochondrial biogenesis in skeletal muscle. J Appl Physiol 2001;90:1137-1157.
Shulman GI: Cellular mechanisms of insulin resistance. J Clin Invest 2000;106:171-176.

23 Melanson EL, Astrup A, Donahoo WT: The relationship between dietary fat and fatty acid intake and body weight, diabetes, and the metabolic syndrome. Ann Nutr Metab 2009;55:229-243.

24 Rasmussen BB, Wolfe RR: Regulation of fatty acid oxidation in skeletal muscle. Annu Rev Nutr 1999; 19:463-484.

25 Simoneau JA, Veerkamp JH, Turcotte LP, Kelley DE: Markers of capacity to utilize fatty acids in human skeletal muscle: relation to insulin resistance and obesity and effects of weight loss. Faseb J 1999;13:2051-2060.

26 Holloway GP, Thrush AB, Heigenhauser GJ, Tandon NN, Dyck DJ, Bonen A, Spriet LL: Skeletal muscle mitochondrial fat/cd36 content and palmitate oxidation are not decreased in obese women. Am J Physiol Endocrinol Metab 2007;292:E17821789.

27 Rogge MM: The role of impaired mitochondrial lipid oxidation in obesity. Biol Res Nurs 2009;10: 356-373.

28 Tudor-Locke C, Bassett DR Jr: How many steps/ day are enough? Preliminary pedometer indices for public health. Sports Med 2004;34:1-8.

29 Tonkonogi M, Fernstrom M, Walsh B, Ji LL, Rooyackers O, Hammarqvist F, Wernerman J, Sahlin K: Reduced oxidative power but unchanged antioxidative capacity in skeletal muscle from aged humans. Pflugers Arch 2003;446:261-269.

-30 Trounce I, Byrne E, Marzuki S: Decline in skeletal muscle mitochondrial respiratory chain function: possible factor in ageing. Lancet 1989;1:637-639.

31 Petersen KF, Befroy D, Dufour S, Dziura J, Ariyan C, Rothman DL, DiPietro L, Cline GW, Shulman GI: Mitochondrial dysfunction in the elderly: possible role in insulin resistance. Science 2003;300: $1140-1142$. 